\title{
Liposil Nanocarriers for Pharmaceutical Applications: Synthesis Innovations
}

\author{
Gonçalves $\mathrm{MC}^{1,2 *}$, Rodrigues $\mathrm{AM}^{3}$, Sabino $\mathrm{A}^{3}$, Corvo $\mathrm{ML}^{4}$ and Martins $\mathrm{MB}^{4}$ \\ ${ }^{1}$ Departamento de Engenharia Química, Instituto Superior Técnico, Universidade Lisboa, Lisboa, Portugal \\ ${ }^{2}$ CQE, Centro de Química Estrutural, Lisboa, Portugal \\ ${ }^{3}$ Departamento de Biongenharia, Instituto Superior Técnico, Universidade de Lisboa, Lisboa, Portugal \\ ${ }^{4}$ iMed.UL, Faculdade de Farmácia da Universidade de Lisboa, Lisboa, Portugal
}

\begin{abstract}
The unique characteristics of liposomes make them interesting vehicles for diagnostic performance. However, when oral administration is envisaged, the adjustment of liposomes surface chemistry is mandatory. Silica-coated liposomes, Liposils for short, become a promising route.

In the present work a novel time-saving process is proposed for liposomes' coating. Two sets of charged liposomes are prepared by lipid hydration and dimensioned through extrusion techniques. The first liposomes set are silica-coated with classical sol-gel Stöber protocol (and used as a reference) while in the second set a modified LaMer process is proved.
\end{abstract}

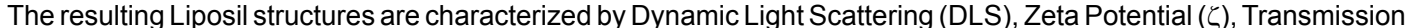
Electron Microscopy (TEM), Fourier Transformed Infrared (FTIR) spectroscopy, Near Infrared (NIR) spectroscopy.

Keywords: Ormosil; Liposil; DLS; Zeta Potential; TEM; FTIR; NIR

\section{Introduction}

Liposomes as self-assembled lipid bi-layers have been widely investigated as biomimetic models of cell membranes, biomimetic conjugates, and as platforms for carrying drugs, gene and image agents due to several intrinsic properties [1-4]. Besides their capacity to load hydrophilic and/or hydrophobic molecules, liposomes i) can penetrate in altered vasculatures due to pathological situations, like in cancer or inflammation, allowing drug accumulation at target sites, ii) are able to drug release at target sites, over a prolonged period which may vary from hours to weeks, and, lastly,

iii) may suffer inner or/and surface chemical modifications to tune drugs biodistribution to achieve active targeting or to enhance chemical/electrostatic bounding to a coating [1-4]. In clinic, for intravenous administration, there are already several pharmaceutical systems where drugs are encapsulated in liposomal structure [5]. However, when oral administration is envisaged and gastrointestinal tract mucus and epithelium barrier need to be overcome, the protection of liposomes from anticipated disruption becomes a promising strategy [6]. The emerging of silica-based drug delivery carriers for oral route administration was the leitmotiv for silica-coating of liposomes, Liposils for short.

The liposome silica-coating is obtained by sol-gel route [7]. Silica precursor molecules hydrolyze, yielding a sol, and then condense, to form a gel able to establish hydrogen-like/electrostatic bounding to the inner liposome structure. The silica-coating helps maintaining the aqueous interior in the liposome-core [6,8-12], and improves the bioavailability of poorly water soluble drugs by enhancing their colloidal stability, retaining, at the same time, the liposome intrinsic advantage as drug carrier. Liposil were reported by Bégu et al. [8] for the first time. Bégu et al. [9] demonstrate that embedded liposomes within a silica-shell maintain their fundamental properties at stomach ( $\mathrm{pH}$ 1.2), while silica hydrolyze at $\mathrm{pH} 7.4$, releasing the encapsulated active drug.

Until now, Liposil structures are difficult to produce due to large time- consuming coating protocols [6,8-12]. Bégu et al. [8] hydrolyze tetraethyl orthosilicate (TEOS) in phosphate-buffered saline solution (PBS), for two days long. The silicate solution is mixed with the liposome suspension, under stirring, at room temperature, for one more day. Then sodium fluoride $(\mathrm{NaF})$ is added, under stirring, for another two days. Liposil structures are separated by filtration (pore size of $200 \mathrm{~nm}$ ). A total of 5 days is needed for the silica coating process. Li et al. [6] add TEOS to a liposome suspension, and let the mixture stir for two days ( 2 days for the liposome coating). Mohanraj et al. [12] propose the faster protocol (1 day), by adding an aqueous suspension of silica nanoparticles (NPs) to an ionically stabilized liposome suspension, and stay stirring overnight.

In the present work two sets of Liposil compositions are studied. In the first Liposil set the organic core is formed by positively charged liposomes of Dipalmitoilfosfatidilcolina (DPPC), Cholesterol (Chol) and Estearilamine (SA), with DPPC:Chol:SA molar ratio of 7:2:1, In the second Liposil set, the core is formed by negatively charged liposomes of 1,2-Dimyristoyl-sn-Glycero-3-Phosphocholine (DMPC) and 1,2-Dimyristoyl-sn-Glycero-3-Phosphoglycerol (DMPG), with DMPC:DMPG molar ratio of 7:3. So far only neutral liposomes were described in literature [6,8-12] when Liposils are concerned.

In the present work anionic and cationic vesicles are proposed for the first time. Further, the liposomes' coating is performed by sol-gel route with hybrid silica. To the best of the authors'knowledge only inorganic-silica coatings have been developed for Liposil structures.

*Corresponding author: Gonçalves MC, Departamento de Engenharia Química, Instituto Superior Técnico, Universidade Lisboa, Lisboa, Portugal, Tel: 351 966622774; E-mail: clara.goncalves@ist.utl.pt

Received: July 17, 2017; Accepted: July 21, 2017; Published: July 28, 2017

Citation: Gonçalves MC, Rodrigues AM, Sabino A, Corvo ML, Martins MB (2017) Liposil Nanocarriers for Pharmaceutical Applications: Synthesis Innovations. Nanomed Nanotechnol 8: 450. doi: 10.4172/2157-7439.1000450

Copyright: (c) 2017 Gonçalves MC, et al. This is an open-access article distributed under the terms of the Creative Commons Attribution License, which permits unrestricted use, distribution, and reproduction in any medium, provided the original author and source are credited. 
In the present work hybrid-silica coatings are proved in both Liposils systems - in situ methyl and amine silica-functionalization is demonstrated. Silica grows on the charged liposome surfaces, forming a core-shell Liposil carrier (liposome-core// functionalized-silica-shell).

Finally the silica growth is carried out by a novel modified timesaving LaMer protocol, which stands on a sharp nucleation process, induced by a strong liquid supersaturation [13]. Only 2 hours are needed for the silica-coating step. To prevent liposome's collapse during coating, LaMer experimental parameters are adjusted (namely $\mathrm{T}, \mathrm{pH}$, and reactional time) in a NPs model.

The synthesized liposome core, and Liposil nanostructures were structurally characterized by Dynamic Light Scattering (DLS), Zeta Potential, Transmission Electron Microscopy (TEM), and Fourier Transform Infrared (FTIR) spectroscopy.

\section{Materials and Methods}

The liposomes are prepared with the following lipids: Dipalmitoilfosfatidilcolina (DPPC), Cholesterol (Chol.) and Estearilamine (SA) (first liposomes set), and 1,2-Dimyristoylsn-Glycero-3-Phosphocholine (DMPC) and 1,2-Dimyristoyl-snGlycero-3- Phosphoglycerol (DMPG) (second liposomes set), all from Avanti Polar Lipids, Inc.

Other chemicals used comprise chloroform (from Panreac Química SA), sodium chloride (from Scharlau Chemie, S.A.), bidistilled water (from Still), absolute ethanol, phosphate saline buffer (PBS buffer $\mathrm{pH}$ 7.4) (from Merck Millipore), ammonia hydroxide (from SigmaAldrich). For silica-coating, silica precursors are (3- Aminopropyl) Triethoxysilane (APTES), Metiltrietoxisilano (MTES) and Tetraethyl Orthosilicate (TEOS) (all from Sigma-Aldrich). Potassium bromide (optical grade, $\geq 99.9 \%$, from Sigma-Aldrich) is used for the FTIR pellets preparation.

\section{Methods}

Liposil synthesis: Liposil synthesis is performed in the two stages: liposomes preparation by Bangham hydration film method [14], follow by dimensioning through extrusion techniques, and liposomes' coating through sol-gel methodology.

The first liposomes set are silica-coated by Stöber [7] methodology while the second set is silica-coated by a modified LaMer [13] protocol. LaMer optimization is performed within silica NPs model.

Liposomes preparation: Liposomes synthesis is based on the Bangham hydration film method [14], immediately followed by dimensioning through extrusion techniques.

For the first liposomes set, $0.0840 \mathrm{~g}$ of DPPC, $0.0120 \mathrm{~g}$ of Chol and $0.0040 \mathrm{~g}$ of SA (DPPC:Chol:SA molar ratio of 7:2:1) are dissolved in chloroform where the lipid film forms by solvent evaporation. The lipid film is hydrated with PBS buffer ( $\mathrm{pH}$ 7.4) ([lipid] $=10 \mathrm{mM}$ ) allowing the formation of lamellar liposomes. During hydration a temperature of $45^{\circ} \mathrm{C}$ was kept constant (lipid transition temperature (Tc) of DPPC is $41^{\circ} \mathrm{C}$ ).

For the second liposomes set, $0.0475 \mathrm{~g}$ of DPMC and $0.0207 \mathrm{~g}$ of DPMG (DPMC:DPMG molar ratio of 7:3) are dissolved in chloroform allowing the lipid film formation by solvent evaporation. The lipid film is hydrated with $10 \mathrm{mM}$ citrate buffer with $0.145 \mathrm{mM} \mathrm{NaCl}(\mathrm{pH} 6)$ ([lipid] $=10 \mathrm{mM}$ ) allowing the formation of multilamellar liposomes.

The two liposomes suspension are sized by extrusion through polycarbonate membranes, with pores ranging from $800 \mathrm{~nm}$ down to $100 \mathrm{~nm}$.

Stober coating (first Liposils series): Stober is far the most common silica growth method used for micro/NPs synthesis and coatings in core-shell nanostructures. The Stöber method is a seed- growth, basic catalyzed sol-gel process, originating fairly monodisperse particles. Ostwald ripening and/or coalescence (promoted by silica dissolution at $\mathrm{pH}$ over 9) are the mechanisms responsible for polydispersity decrease over time, with $\mathrm{R}^{-1}$, being $\mathrm{R}$ the particle radius.

In the first liposomes' set the hybrid-silica-coating is obtained through the classical Stöber protocol, based on Bégu et al. [8]. TEOS and MTES are firstly hydrolyzed in PBS (pH 7.4) for two days, at $40^{\circ} \mathrm{C}$. Then the silicate solution (SSS, for short) is added to the liposomes suspension (silica precursor: lipid 8:1 molar ratio) and kept under moderate stirring $(<60 \mathrm{rpm})$ for another day. Finally $\mathrm{NaF}$ is added to the mixture, at ambient temperature and protected from light, and kept under stirring for another $48 \mathrm{~h}$.

LaMer coating (second Liposils series) LaMer optimization (NPs model): When monodisperse NPs are designed, sharp nucleation is needed and LaMer protocol emerges as an interesting option. Nevertheless its high $\mathrm{pH}(\sim 11)$ and process temperature $\left(\sim 55^{\circ} \mathrm{C}\right)$ hinder its use when liposomes' coating is regarded. To adapt the LaMer protocol to the liposomes' coating several parameters are optimized in NPs model. Amine-functionalized-silica NPs model are used for optimizing: 1) temperature, 2) $\mathrm{pH}$ and 3) reactional time.

Temperature: To avoid liposome disruption the temperature of $20^{\circ} \mathrm{C}$ was set for the sol-gel synthesis. (DMPC and DMPG are used as case study for Liposil construction. Tc of DMPC and DMPG are $24^{\circ} \mathrm{C}$ and $23^{\circ} \mathrm{C}$, respectively).

$p H$ : To harmonize the sol-gel process with the $\mathrm{pH}$ of liposomes synthesis/storage buffer solution $(10 \mathrm{mM}$ citrate buffer with 0.145 $\mathrm{mM} \mathrm{NaCl}(\mathrm{pH}$ 6)) LaMer synthesis is firstly performed at $\mathrm{pH} 7$ $\left(\mathrm{pH}=7, \mathrm{~T}=20^{\circ} \mathrm{C}\right)$. Amine-functionalize-silica NPs of $1908 \pm 281 \mathrm{~nm}$ hydrodynamic diameter are obtained (due to aggregation), ruling out this $\mathrm{pH} / \mathrm{T}$ set value. Performing LaMer synthesis at $\mathrm{pH} 9\left(\mathrm{~T}=20^{\circ} \mathrm{C}\right)$, amine-functionalize-silica NPs of $136.0 \pm 0.6 \mathrm{~nm}$ hydrodynamic diameter are successfully produced (due to silica dissolution over $\mathrm{pH}$ 7 [7].

Reactional time (time interval between the addition of silica precursors to the liposome suspension and centrifuge unit operation) is kept constant and equal to $1 \mathrm{~h}$.

Reactional time: To study the silica growth process two reactional times were proved $-1 \mathrm{~h}$ and $2 \mathrm{~h}$ (at preset $\mathrm{pH}=9$ and $\mathrm{T}=20^{\circ} \mathrm{C}$ ). $136.0 \pm$ $0.6 \mathrm{~nm}$ and $761.0 \pm 74.1 \mathrm{~nm}$ hydrodynamic diameters were obtained, respectively. Based on NPs model, LaMer selected experimental conditions are: $\mathrm{T}=20^{\circ} \mathrm{C}, \mathrm{pH}=9$ and $1 \mathrm{~h}$ reactional time.

LaMer coating of liposomes: A modified LaMer protocol is ready for coating liposomes structures (liposome suspension (lipid=10 mM).

Briefly, 0.422 up to $3.80 \mathrm{~mL}$ of liposomes (DMPC:DMPG at 7:3 molar ratio) are mixed with silica-precursors' solution (with a constant value of TEOS, $3.80 \mathrm{~mL}$, and APTES, $0.422 \mathrm{~mL}$ ) and water to obtain a final volume of $4.5 \mathrm{~mL}$. The range of volume ratio [Liposomes:TEOS] were 1:9, 1:4,1:2.3, 1:1.5, 1:1. The maximization of the liposomes concentration in basic environment is the main goal at this stage.

Each of the previously described $4.5 \mathrm{~mL}$ suspensions are dispersed 
under stirring in a solution of $89.4 \mathrm{~mL}$ of ethanol, $2.3 \mathrm{~mL}$ of ammonia hydroxide.

The resulting mixture is maintained at moderate magnetic agitation $(350 \mathrm{rpm})$ for 1 hour, at room temperature. After this period, the suspension is centrifuged $\left(8000 \mathrm{rpm}, 23^{\circ} \mathrm{C}, 15 \mathrm{~min}\right)$ and the supernatant removed. The centrifuged product is re- suspended in ethanol or water, sonicated for 15 minutes and centrifuged again. The washing process is repeated six times - with ethanol and distilled water. The final product is re-suspended in distilled water and stored in the fridge $\left(\sim 4^{\circ} \mathrm{C}\right)$.

\section{Structural characterization}

Dinamic light scattering and zeta potential: Dinamic Light Scattering (DLS) determines the hydrodynamic diameter and its dispersity, and was performed with Malvern Zetasizer Nano S. Zeta Potential $(\zeta)$ assesses colloidal stability and is measured with Malvern Nano. Both DLS and $\zeta$ analyses follow a similar procedure: introduction of $10 \mu \mathrm{L}$ ofultra-sonicated sample ( 2 minutes) and $1000 \mu \mathrm{L}$ of Milli-Q water in each cell. The data is analyzed with Zetasizer Series software.

TTEM: TEM show NPs, liposomes and Liposils' morphology and size. TEM analysis is performed on a transmission electronic microscope (Hitachi 8100), with LaB6 filament, and accelerating voltage of $100 \mathrm{kV}$. A drop of the colloidal suspension is deposited in a copper grid and analyzed after solvent evaporation. A negative stain with phosphotungstic acid (PTA) was performed as described by [15].

FTIR and NIR spectroscopies: FTIR spectra are obtained using a Nicolet 5700, Thermo Electron Corporation, in transmission mode in the medium infrared (IR) range, with 512 scans, and $4 \mathrm{~cm}^{-1}$ resolution. $\mathrm{KBr}$ pellet method is used. The pellets of $200 \mathrm{mg}$ each are pressed in a manual hydraulic press (from Specac). A drop of each sample is deposited between two $\mathrm{KBr}$ pellets. Two $\mathrm{KBr}$ pellets are used as background in FTIR measurements. Near Infrared Spectroscopy (NIR) spectra are obtained using the FTLA 2000- 160 spectrometer, ABB Analytical, in transmittance mode, with $32 \mathrm{scans}$, and $6 \mathrm{~cm}^{-1}$ resolution. Water is used as background in NIR measurements. The clustering of the samples by their nature - liposomes, Ormosil NPs and Liposils is obtained through application of chemometric techniques in the FTIR and NIR spectrums. After collect FTIR and NIR spectra, for multivariate analysis, the data is analyzed at SIMCA-P $\mathrm{P}^{\infty}$ software, trial version 13.0.2, Umetrics. The spectra is filtered with Savitzky-Golay filter (with 11 points), and first order derivative. The application of this method allows the preservation of the characteristics of distribution increases the resolution and eliminates interference caused by the particle size [16].

\section{Results and Discussion of Liposil Synthesis}

Liposil compositions are synthesized in two stages: a lipid-core preparation followed by sol-gel hybrid-silica coating. Concerning liposomes core, two different sets are studied: first series comprises DPPC:Chol:SA (with molar ratio of $7: 2: 1$ ) and the second series DMPC:DMPG (with a molar ratio of 7:3). In the first liposomes series hybrid-silica-coating growths through Stöber, while in the second a modified LaMer protocol is used. LaMer optimization is based on feedback model supported on silica NPs.

\section{Liposomes preparation}

In the present work two different liposomes sets are prepared: the first one comprises a mixture of DPPC:Chol:SA with a molar ratio of 7:2:1, while the second one consists of a lipid mixture of two different phospholipids DMPC:DMPG at 7:3 molar ratio. Liposomes are extruded through several polycarbonate membranes Table 1 illustrates DLS, polydispersity and $\zeta$ of each liposomes set.

\section{Stober coating (first Liposils series)}

Stober in situ methyl-functionalized-silica shells growth over the inner core (DPPC:Chol:SA) producing the first Liposils series (Figures 1 and 2). Sol-gel hydrolysis was performed without using alcohol as co-solvent. The alcohol formed during condensation, as sub-product, is enough to promote the solubility between TEOS and water, allowing the progress of the hydrolysis reaction. The exclusion of alcohol at this stage boosts the number of sol-gel applications in biotechnology (due to the sensitivity of some biological systems to the presence of alcohol).

\section{LaMer coating (second Liposil series)}

LaMer optimization: LaMer in situ amine-functionalized-silica shells growth over the inner core (DMPC:DMPG) producing the second Liposils series.

Base on NPs model, LaMer protocol was optimized aiming liposomes' silica coating. Process temperature, $\mathrm{pH}$ and reactional times are studied to harmonize the sol-gel protocol with the liposomes synthesis/storage buffer conditions more severe in the second liposomes set (liposomes' Tc: $24^{\circ}$ for DMPC, $23^{\circ} \mathrm{C}$ for DMPG against $41^{\circ} \mathrm{C}$ for DPPC).

Temperature is a determinant parameter in any kinetic process. $55^{\circ} \mathrm{C}$ is the temperature commonly used in LaMer silica NPs synthesis. In the present study the liposomes' Tc $\left(24^{\circ}\right.$ for DMPC and $23^{\circ} \mathrm{C}$ for DMPG) limits the experimental temperature. Outstripping Tc compromises the lipid membrane fluidity, ruling out the silica growth over the liposomes core. In the present work, temperature is set at $20^{\circ} \mathrm{C}$.

$\mathrm{pH}$ is a paramount parameter. In the sol-gel process $\mathrm{pH}$ controls both the hydrolysis and condensation mechanisms, and determines the final product morphology. So far LaMer protocol was used at $\mathrm{pH} 11$ (along with $\mathrm{T}=55^{\circ} \mathrm{C}$ ).

Sharp monodispersed amine-functionalized silica NPs were synthesized at $\mathrm{pH} 9\left(\right.$ at $\mathrm{T}=20^{\circ} \mathrm{C}$ ) where the NPs monodispersity depends on a strong supersaturation followed by burst nucleation. Further, in silica sol-gel process, silica solubility, Ostwald ripening and aggregation mechanisms all decrease with $\mathrm{pH}$.

Time is a central parameter in kinetics. NPs keep growing until one (or both) reactant(s) are consumed. Two different reactional times are tested - 1 and 2 hours (h). Hydrodynamic diameter, of the silica NPs synthesized with $1 \mathrm{~h}$ and $2 \mathrm{~h}$ reactional times, are shown in Table 2. NPs obtained with 1 hour reactional time were also observed by TEM (Figure 3 ).

\begin{tabular}{|l|c|c|c|}
\hline & DPPC:Chol:SA (7:2:1) 200 nm (EPM) & DMPC:DMPG (7:3) 100 nm (EPM) & DMPC:DMPG (7:3) 200 nm (EPM) \\
\hline DLS $(\mu \mathrm{m})$ & $0.176 \pm 0.002$ & $0.150 \pm 0.010$ \\
\hline DLS Polydispersity $(\mu \mathrm{m})$ & 0.002 & 0.076 & $0.183 \pm 0.920$ \\
\hline Zeta potential $(\mathrm{mV})$ & 30 & -30 \\
\hline
\end{tabular}

EPM: Extrusion Using Polycarbonate Membranes.

Table 1: Hydrodynamic diameter, polydispersity and zeta potential for liposomes DPPC:Chol:SA (7:2:1) (first series) and DMPC:DMPG at (7:3) (second series). 
Citation: Gonçalves MC, Rodrigues AM, Sabino A, Corvo ML, Martins MB (2017) Liposil Nanocarriers for Pharmaceutical Applications: Synthesis Innovations. J Nanomed Nanotechnol 8: 450. doi: 10.4172/2157-7439.1000450

a)

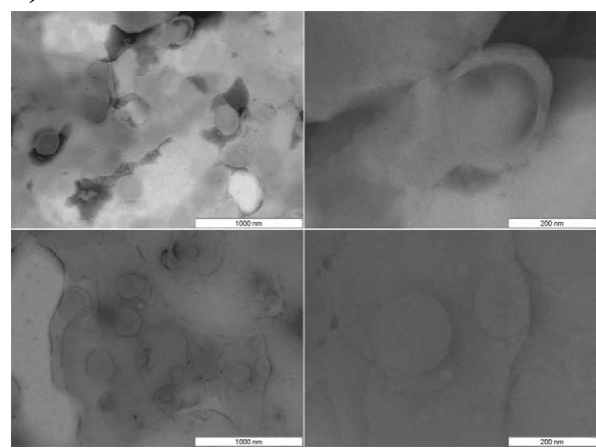

b)

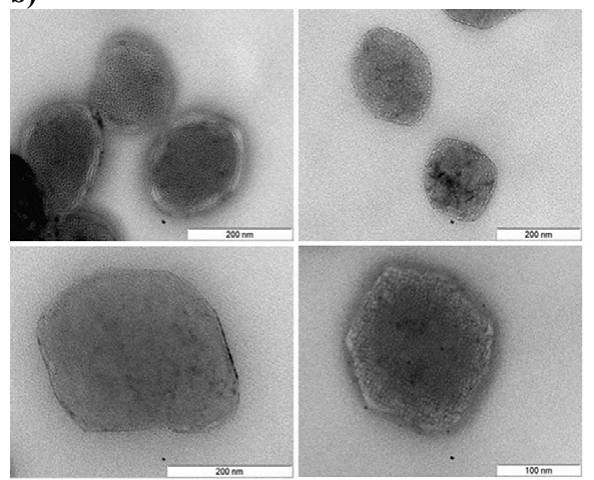

Figure 1: TEM images of two sets of liposomes with negative stain (PTA): (a) DPPC:Chol:SA (7:2:1) (first series) and (b) DMPC:DMPG (7:3) (second series).

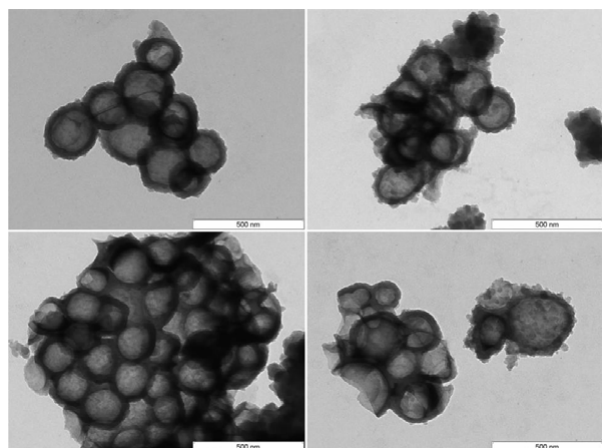

Figure 2: TEM images of first Liposil series (DPPC:Chol:SA (7:2:1) core) with negative stain (PTA), synthesized by Stöber.

\begin{tabular}{|c|c|}
\hline Reactional time & Hydrodynamic diameter $(\mathbf{n m})$ \\
\hline $1 \mathrm{~h}$ & $136 \pm 0.61$ \\
\hline $2 \mathrm{~h}$ & $761 \pm 74.1$ \\
\hline
\end{tabular}

Table 2: Hydrodynamic diameter of silica NPs synthesized at $\mathrm{pH} 9,20^{\circ} \mathrm{C}$, within $1 \mathrm{~h}$ and $2 \mathrm{~h}$ of reactional time.

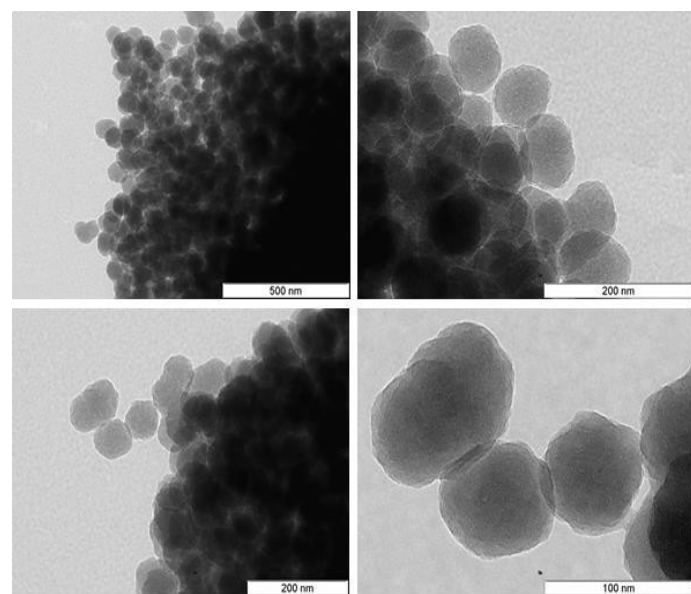

Figure 3: TEM images of silica NPs synthetized by LaMer protocol $\left(20^{\circ} \mathrm{C}, \mathrm{pH}\right.$ 9 and $1 \mathrm{~h}$ reactional time).

LaMer modification: In Liposil structures the ratio between lipid:silica is a relevant parameter. So, changing the volume of liposomes and keeping constant the silica precursors' volumes $(3.8 \mathrm{~mL}$ of TEOS and $0.422 \mathrm{~mL}$ of APTES) several volume ratio liposomes:TEOS were tested, in the range 1:9 up to 1:1 in this study (Table 3). Zeta potential, hydrodynamic diameter and polydispersity are studied targeting nanocarriers' production and presented in Table 3.

All Liposil compositions exhibited kinetic colloidal stability $(\zeta \leq-30$ $\mathrm{mV}$ ) overtime (even after 1 year storage) making them suitable for industrial use. The different Liposil preparations were observed by TEM as is illustrated in Figure 4.

The morphology of the Liposil particles observed by TEM evidence that the core-shell structure is dependent of the ratio liposomes:TEOS. A limit value of liposomes:TEOS ratio 1:1 is needed to allow silica growth over the inner liposomes core. Liposil particles A and B do not evidence a core-shell structure. Liposil C and D slightly evidence a core structure. Liposil E evidence a core shell structure where Liposils displayed the presence of spheres mostly grouped in clusters, with individual Liposils retaining their perfectly spherical shape and wall integrity. The aggregation appears due to sample drying step which is inherent of TEM procedure.

By TEM and DLS results, it was concluded that the Liposils size increases with increased volume of liposomes during Liposil synthesis, evidencing that the liposomes are the core of the nanostructures. The increase in particle size going from liposomes to Liposils occurred during the condensation phase (after addition of hydrolyzed TEOS) due to the formation of the silica shell.

\section{Characterization}

The FTIR spectroscopy allows fingerprint of the structures (Table 4).

\begin{tabular}{|c|c|c|c|c|c|c|}
\hline Liposil & Liposomes mL & TEOS mL & APTES $\mathrm{mL}$ & Diameter* $(\mathrm{nm})$ & Polydispe rsity (nm) & Zeta potential $(\mathrm{mV})$ \\
\hline$A(1: 9)$ & 0.422 & 3.80 & 0.422 & $161.0 \pm 4.5$ & 0.241 & $-39.10 \pm 1.61$ \\
\hline$B(1: 4)$ & 0.950 & 3.80 & 0.422 & $180.0 \pm 1.7$ & 0.214 & $-36.40 \pm 1.57$ \\
\hline$C(1: 2.3)$ & 1.630 & 3.80 & 0.422 & $276.0 \pm 6.1$ & 0.237 & $-31.60 \pm 3.13$ \\
\hline$D(1: 1.5)$ & 2.530 & 3.80 & 0.422 & $708.0 \pm 55$ & 0.570 & $-41.30 \pm 0.44$ \\
\hline$E(1: 1)$ & 3.800 & 3.80 & 0.422 & $807.0 \pm 39$ & 0.518 & $-37.90 \pm 0.80$ \\
\hline
\end{tabular}

*Diameter by DLS.

Table 3: Effect of the volume ratio liposomes:TEOS in the diameter, polydispersity and zeta potential of Liposil samples (second series). 
Citation: Gonçalves MC, Rodrigues AM, Sabino A, Corvo ML, Martins MB (2017) Liposil Nanocarriers for Pharmaceutical Applications: Synthesis Innovations. J Nanomed Nanotechnol 8: 450. doi: 10.4172/2157-7439.1000450
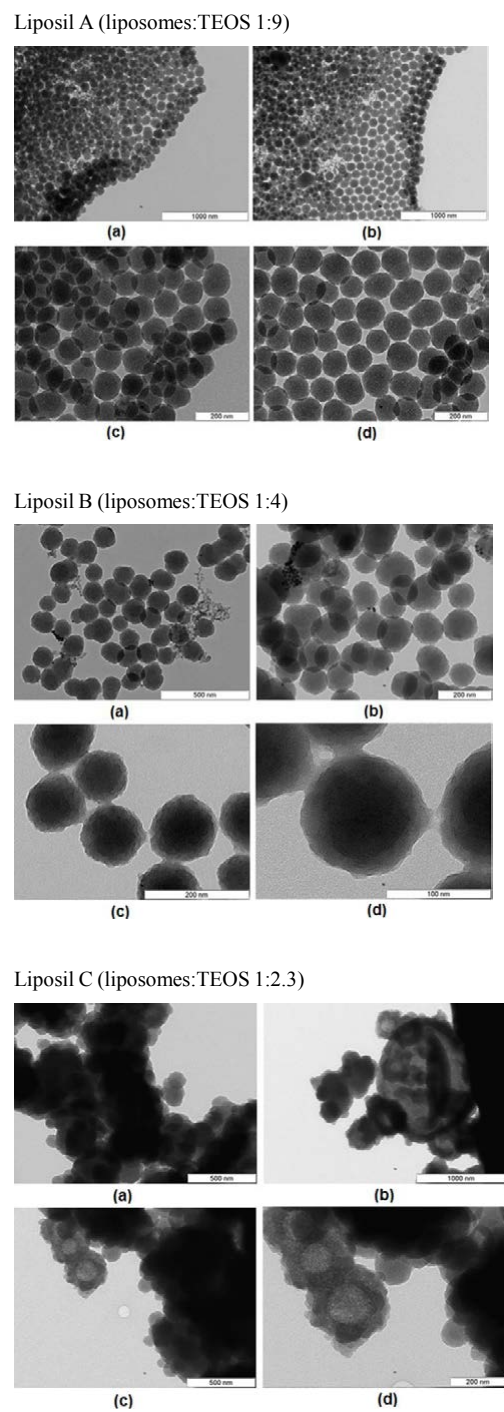

Liposil D (1:1.5)

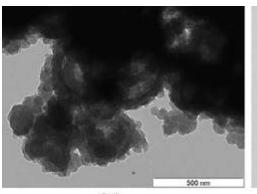

(a)

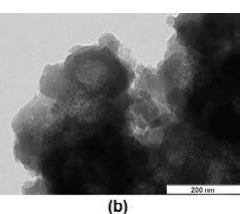

(b)

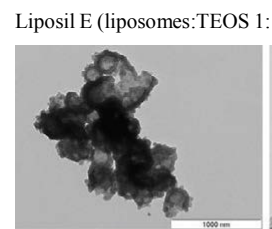

(a)

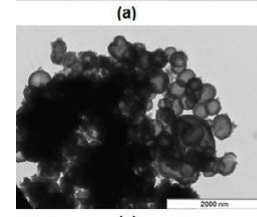

(c)
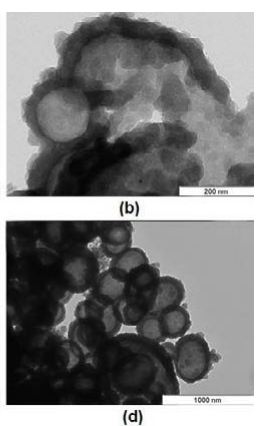

Figure 4: TEM images of second Liposils series (DMPC:DMPG core) with negative stain (PTA), synthesized by LaMer (the correspondent liposomes: TEOS ratio is identified for each panel).

\begin{tabular}{|l|l|}
\hline $1200-1000 \mathrm{~cm}^{-1} \rightarrow \mathrm{Si}-\mathrm{O}$ & $\begin{array}{l}\text { Proves the silica network in ormosil NPs and } \\
\text { Liposil structures (Ormosil coating) }\end{array}$ \\
\hline $\begin{array}{l}1210-1140 \rightarrow \mathrm{P}=\mathrm{O} 2420- \\
2270 \mathrm{~cm}^{-1} \rightarrow \mathrm{P}-\mathrm{O}\end{array}$ & $\begin{array}{l}\text { The P=O and P-O bonds (characteristic of the } \\
\text { lipids present in DMPC and DMPG liposomes) } \\
\text { proves their presence in Liposils structures }\end{array}$ \\
\hline $1700-1550 \mathrm{~cm}^{-1} \rightarrow \mathrm{H}-\mathrm{O}-\mathrm{H}$ & $\begin{array}{l}\text { Confirms the presence of water molecules within } \\
\text { and on the surface of Liposils (demonstrating their } \\
\text { hydrophilic nature) }\end{array}$ \\
\hline $3000-2850 \mathrm{~cm}^{-1} \rightarrow \mathrm{C}-\mathrm{H}$. & $\begin{array}{l}\text { Their absence in Ormosil NPs spectrum indicates } \\
\text { that are no residues of non-hydrolyzed alkoxy } \\
\text { groups from precursor molecules. In Liposils prove } \\
\text { the existence of a liposomal core, since the } \\
\text { C-H bonds from aliphatic compounds are present } \\
\text { in lipids such as DMPC and DMPG }\end{array}$ \\
\hline $3500-3300 \mathrm{~cm}^{-1} \rightarrow \mathrm{O}-\mathrm{H}$, & $\begin{array}{l}\text { Liposil nanostructures were dispersed in aqueous } \\
\text { medium, whereby the OH peak is the one with } \\
\text { highest intensity, sensing the presence of } \\
\text { adsorbed water molecules. The Si-O peak proves } \\
\text { the silica network in Ormosil NPs and Liposils } \\
\text { structures (Ormosil coating) } \\
\mathrm{N}-\mathrm{H} . \mathrm{H} \text { proves the existence of the hybrid silica in } \\
\text { Ormosil NPs and Liposils (coating) }\end{array}$ \\
\hline
\end{tabular}

Table 4: Representative IR ranges present in Liposil.

Liposomes, silica and amine- or methyl- groups have been identified. Figure 5 illustrates the some of the Liposils structures of the second set. Analyzing the spectra present in Figure 5, it is observed that the Liposils C, D and E spectra are similar to the liposomes spectrum, justified by the largest liposomes/silica ratio.

NIR spectroscopy is used, primarily, for quantitative analysis and not for the identification of specimens. Given that NIR absorption bands present overlaps and, for this reason, the analytical information is multivariate, being necessary to resort to statistical processing chemometrics [17]. However, the use of chemometrics techniques was also subjected to FTIR spectrum.

The FTIR and NIR spectrums were pretreated with SavitzkyGolay filter with 11 points and $1^{\text {st }}$ derivate, in SIMCA-P ${ }^{\infty}$ software. This pretreatment enables characteristics of distribution preservation, increases the resolution and eliminates interference caused by the particle size.

Principal Components analysis shows that both models presents are good (R2 close to 1 ) and present good predictivity (Q2>0.5), but due to the low samples number they do not present statistical significance.

Scores Plot of the samples analyzed by FTIR and NIR (Figures 6 and 7) show that, in general, the model can distinct three groups Ormosil, liposomes and Liposil - except for Liposils A and B samples that appear closer to the Ormosil sample. Given that Liposils A and $\mathrm{B}$ are composed by a lower liposomes volume and morphologically, exhibit greater similarity to the Ormosil NPs, this clustering is justified. This cluster was expected by analyzing the FTIR spectrum presented in Figure 4.

\section{Conclusion}

The goal of this work was the design, the synthesis and the characterization of hybrid nanostructures named Liposil, i.e. liposomes coated with an amorphous silica/ Ormosil shell.

Two different Liposil series are synthesized and characterized. Liposome composition and the Ormosil synthesis optimization were performed.

In the first Liposil series a classical Stöber method is used for the 


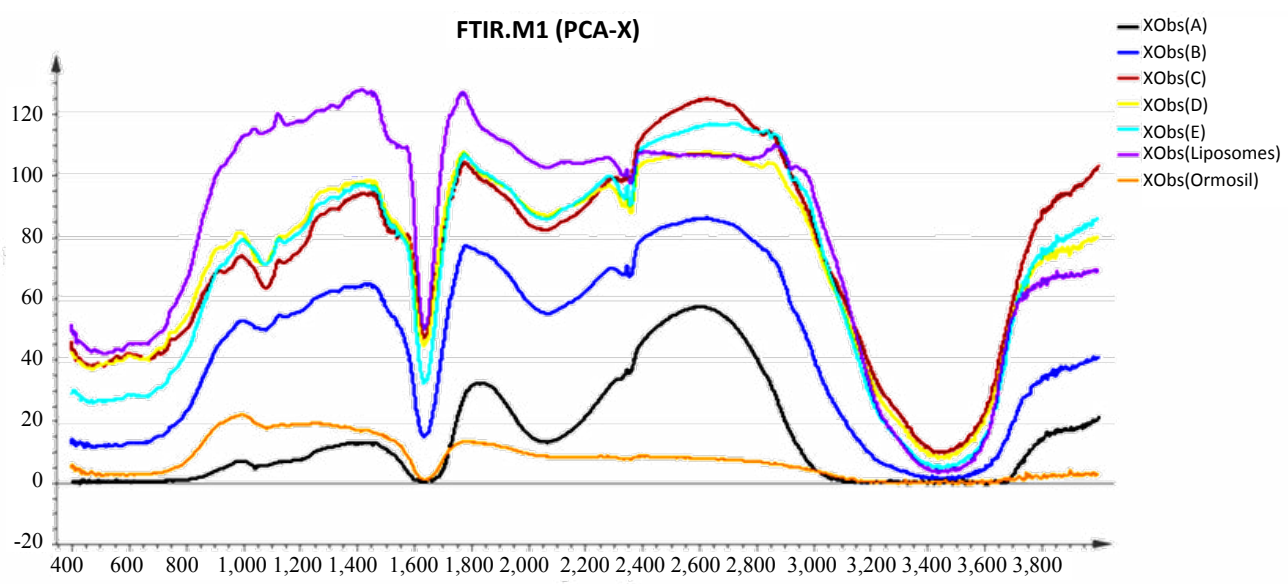

Figure 5: FTIR spectrums of liposomes, silica NPs and Liposils (A, B, C, D, E, second series) structures.

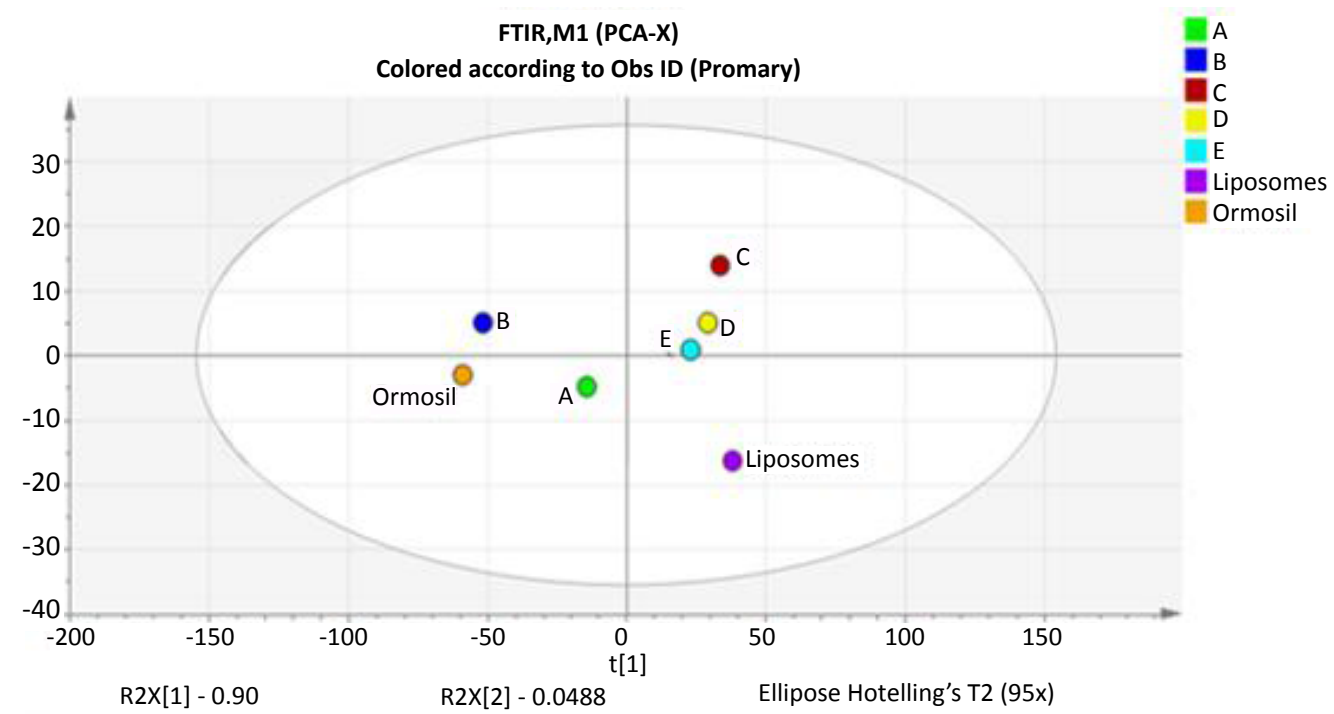

Figure 6: Scores Plot Analysis of liposomes, silica NPs and Liposils (A, B, C, D, E second series) samples analyzed by FTIR.

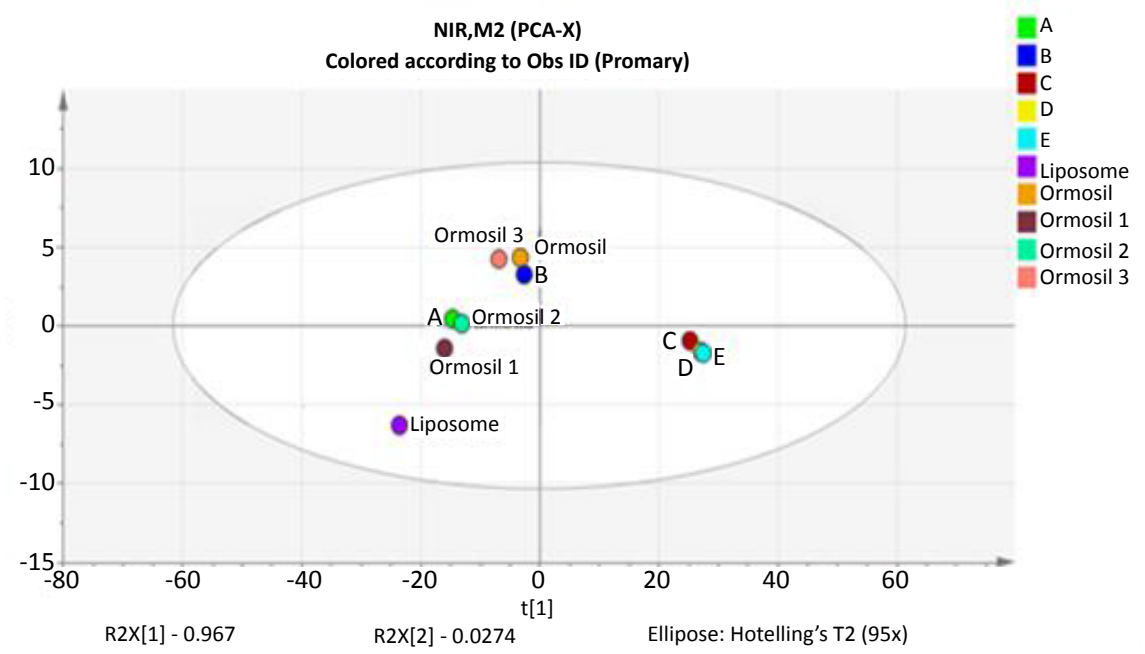

Figure 7: Scores Plot Analysis of Scores Plot Analysis of liposomes, silica NPs and Liposils (A, B, C, D, E second series) samples analyzed by NIR. 
Citation: Gonçalves MC, Rodrigues AM, Sabino A, Corvo ML, Martins MB (2017) Liposil Nanocarriers for Pharmaceutical Applications: Synthesis Innovations. J Nanomed Nanotechnol 8: 450. doi: 10.4172/2157-7439.1000450

silica growth over the inner liposomes core (DPPC:Chol:SA). In the second Liposil series, a LaMer protocol is developed and optimized, aiming harmonization of liposomes (DMPC: DMPG) stability and solgel process.

For the second Liposil series the process temperature is set at $20^{\circ} \mathrm{C}$, to respect the lipid transition phase temperatures of both DMPC and DMPG lipids, $24^{\circ}$ and $23^{\circ} \mathrm{C}$, respectively. Secondly, a $\mathrm{pH}$ of 9 is selected to harmonize the sol-gel process with the liposomes synthesis/ storage buffer solution. Additionally reactional time and $\zeta$ are studied to evaluate the process scalability.

DLS and TEM characterization methods allow the selection of parameters for optimizing Liposils synthesis. This indicates that the liposomes serve as a nanostructures core. According to TEM characterization Liposil nanostructure evidence a liposomes core and a hybrid silica shell, which present silica wall thickness in agreement with the expected values were observed. TEM analysis showed particle aggregation due to the drying step inherent of TEM procedure. Besides size, shape and composition, we may conclude that the silica shell provides liposomes properties maintenance.

Zeta potential analysis confirms Liposils colloidal stability $(\zeta \leq-30$ $\mathrm{mV}$ ) making them good candidates to carry hydrophilic, hydrophobic or amphiphilic drugs.

FTIR analysis evidences the presence of hybrid silica network and lipid groups - DMPC and DMPG - proving that liposomes are efficiently coated by Ormosil shell. FTIR and NIR principal components analysis (PCA) allowed distinguishing three groups - liposomes, Ormosil and Liposil - where Liposil are closer to Ormosil sample, justified by the composition and morphology of these Liposils. Despite the model created, it would be necessary to insert a greater number of samples to increase the statistical significance.

This study provides the basis for future study of Liposil for drug(s) encapsulation. Cryo-TEM characterization is one characterization technique recommended for future work.

\section{References}

1. Wang B, Hu L, Siahaan TJ, Wang G (2016) Liposomes as Drug Delivery vehicles. Wiley Series in Drug Discovery and Development, Wiley New Jersey.
2. Pattni BS, Chupin VV, Torchilin VP (2015) New developments in liposomal drug delivery. Chemical reviews 115: 10938-10966.

3. Allen TM, Cullis PR (2013) Liposomal drug delivery systems: from concept to clinical applications. Advanced Drug Delivery Reviews 65: 36-48.

4. De Araújo Lopes SC, dos Santos Giuberti C, Rocha TG, dos Santos Ferreira D, Leite EA, et al. (2013) Liposomes as carriers of anticancer drugs. In Cancer Treatment-Conventional and Innovative Approaches.

5. Barenholz Y (2012) Doxil the first FDA-approved nano-drug from an idea to a product. Laboratory of Membrane and Liposome Research 335-396.

6. Li C, Zhang Y, Su T, Feng L, Long Y, et al. (2012) Silica-coated flexible liposomes as a nanohybrid delivery system for enhanced oral bioavailability of curcumin. International Journal of Nanomedicine 7: 5995-6002.

7. Brinker CJ, Scherer GW (2013) Sol-gel science: the physics and chemistry of sol-gel processing. Academic press 2: 97-145.

8. Bégu S, Girod S, Lerner DA, Jardiller N, Tourné-Péteilh C, et al. (2004) Characterization of a phospholipid bilayer entrapped into non-porous silica nanospheres. Journal of Materials Chemistry 14: 1316-1320.

9. Bégu S, Pouëssel AA, Lerner DA, Tourné-Péteilh C, Devoisselle JM (2007) Liposil, a promising composite material for drug storage and release. Journal of Controlled Release 118: 1-6.

10. Shen S, Kendall E, Oliver A, Ngassam V, Hu D, et al. (2011) Liposil-supported lipid bilayers as a hybrid platform for drug delivery. Soft Matter 7: 1001-1005.

11. Folliet N, Roiland C, Bégu S, Aubert A, Mineva T, et al. (2011) Investigation of the interface in silica-encapsulated liposomes by combining solid state NMR and first principles calculations. Journal of the American Chemical Society 133: 16815-16827.

12. Mohanraj VJ, Barnes TJ, Prestidge CA (2010) Silica nanoparticle coated liposomes: a new type of hybrid nanocapsule for proteins. International Journal of Pharmaceutics 392: 285-293.

13. Carvalho A, Domingues I, Gonçalves MC (2015) Core-shell superparamagnetic nanoparticles with interesting properties as contrast agents for MRI. Materials Chemistry and Physics 168: 42- 49

14. Bangham AD (1978) Properties and uses of lipid vesicles: an overview. Annals of the New York Academy of Sciences 308: 2-7.

15. Faria MR, Cruz MM, Gonçalves MC, Carvalho A, Feio G, et al. (2013) Synthesis and characterization of magnetoliposomes for MRI contrast enhancement International Journal of Pharmaceutics 446: 183-190.

16. Reich G (2005) Near-infrared spectroscopy and imaging: basic principles and pharmaceutical applications. Advanced Drug Delivery Reviews 57: 1109-1143.

17. Jaganathan H, Godin B (2012) Biocompatibility assessment of Si-based nanoand micro-particles. Advanced Drug Delivery Reviews 64: 1800-1819. 